\title{
Effect of overexpression of the 5-HT7 receptor gene on behavior and brain serotonin system in ASC mice with predisposition to depressive-like behavior
}

\author{
Baraboshkina I.A. \\ ICG SB RAS, Novosibirsk, Russia \\ irina.10.24@yandex.ru \\ Antonov E.V. \\ ICG SB RAS, Novosibirsk, Russia \\ a.yegor.v@gmail.com
}

\author{
Bazovkina D.V. \\ ICG SB RAS, Novosibirsk, Russia \\ daryabazovkina@gmail.com \\ Kulikova E.A. \\ ICG SB RAS, Novosibirsk, Russia \\ kulikova.elisa@gmail.com
}

\author{
Ilchibaeva T.V. \\ ICG SB RAS, Novosibirsk, Russia \\ rbicehok@mail.ru \\ Naumenko V.S. \\ ICG SB RAS, Novosibirsk, Russia \\ naumenko2002@gmail.com
}

\begin{abstract}
The serotonin (5-HT) system of the brain plays an important role in controlling various behaviors due to the wide variety of serotonin receptors. The 5-HT7 receptor is of great interest because it is involved in the pathogenesis of depressive disorders. Mice of ASC (Antidepressant Sensitive Catalepsy) line with genetic predisposition to depressive-like behavior were obtained in the Laboratory of Behavioral Neurogenomics of ICG SB RAS (Novosibirsk). In this work, the effect of adenoassociated virus (AAV)-mediated overexpression of the 5-HT7 receptor gene in the midbrain on the behavior and brain serotonin system in ASC mice was comprehensively studied. The ASC mice with overexpression of 5-HT7 receptor gene showed the decrease in depressive-like behavior in the forced swim test compared to control group. The introduction of a vector construct with the 5-HT7 receptor gene affected the expression of the gene encoding the 5-HT7 receptor itself, only in the midbrain. The overexpression of 5-HT7 receptor gene in midbrain led to an increase in the serotonin metabolism index in the cortex, hippocampus, and midbrain of mice, without affecting serotonin levels. The results indicate the effect of overexpression of the 5-HT7 receptor gene on the behavior and serotonin system of the brain in ASC mice with a genetic predisposition to depressive-like behavior.
\end{abstract}

Keywords - serotonin brain system; 5-HT7 receptor; mice; depressive-like behavior; vector construct; overexpression; gene

\section{Introduction}

Currently, an increasing number of people suffer from various psychopathologies. According to World Health Organization, about $25 \%$ of the global population suffer from depressive disorders. Thus, at the moment, the problem of finding therapeutic agents and antidepressants for the treatment of diseases of this type is very urgent. It is known that currently existing antidepressants do not have high enough effectiveness, and also have a number of side effects. Thus, when creating new medicines in this direction, first of all, the task is to increase their effectiveness and reduce the possible side effects of their use.

The serotonin system of the brain plays an important role in controlling various behaviors, including pathological ones, due to the large number of types of serotonin receptors and their wide distribution in the brain [1]. The 5-HT1A receptor is involved in mechanisms of response to stress, depression, anxiety, and aggression. Also, 5-HT1A receptors located presynaptically regulate the functional activity of the serotonin system of the brain by the mechanism of negative feedback [2]. 5-HT2A receptors are involved in the mechanisms of development of a number of mental disorders, including depression and schizophrenia, and are also an important link in the mechanisms of action of antidepressant agents [3].

The 5-HT7 receptor is of great interest and has been studied relatively recently. The 5-HT7 receptor is associated with various physiological and pathophysiological processes, including circadian rhythms, thermoregulation, and depression [4]. It was found that it can influence the regulation of the serotonin system of the brain, so its study is a promising direction today. Modern literature suggests that there is a dependence of the presynaptic 5-HT1A receptor on 5-HT7 receptors, which, forming dimer complexes with it, lead to functional inactivation of 5-HT1A receptor [2]. This is a completely new and crucial role of the 5-HT7 receptor and its interaction with the 5-HT1A receptor allows us to take a new look at the problem of the mechanism of depression and the action of antidepressants of the serotonin reuptake inhibitor group.

It is important to select adequate animal models for the study of psychopathological conditions. Mice of ASC (Antidepressant Sensitive Catalepsy) line obtained in the Institute of Cytology and Genetics SB RAS (Novosibirsk) demonstrate pronounced depressive-like behavior and dysfunctions of immune system, which is also observed in depressed patients. Thus, ASC mice meet all the necessary criteria for a laboratory model of depression.

Manipulating the expression of certain genes is a promising area of study and treatment of psychopathologies. This approach is carried out using vectors that ensure delivery of genetic material to the cells of the body and its expression. Recombinant adenoassociated viruses are one of the most promising delivery vectors in gene therapy and neurobiology. Their most important advantages are: non-pathogenicity, low immunogenicity, tropicity to most cells and tissues, as well as high efficiency of transduction and long time of gene expression in vivo without embedding in the host genome.

Purpose of work was to study the effect of overexpression of the 5-HT7 receptor gene in the midbrain on the behavior and brain serotonin system in mice with a predisposition to depressive-like behavior.

In accordance with this, the following tasks were formulated:

1) to investigate the effect of overexpression of the 5-HT7 receptor gene on behavior in the open field and forced swim tests in ASC mice;

2) to study the effect of overexpression of the 5-HT7 receptor gene on the level of mRNA genes encoding key 
elements of the serotonin system in various brain structures of ASC mice;

3) to evaluate the effect of overexpression of the 5-HT7 receptor gene on level of 5-HT and and their metabolite 5HIAA in different brain structures of ASC mice.

\section{Materials and Methods}

Experiments were conducted on adult three-month-old male mice of the ASC line (Antidepressant sensitive catalepsy), $(n=30)$ with genetic predisposition to depressivelike behavior. This line was obtained in the Laboratory of Behavioral Neurogenomics of Institute of Cytology and Genetics (Novosibirsk) by selection for high predisposition to catalepsy from the population of backcrosses between the cataleptic CBA and noncataleptic AKR strains.

The animals were divided into 3 groups: the first group of mice (Control) received a buffer into the midbrain (a solvent for the vector construct), the second group of mice (AAV_HTR7_eGFP) received the actual construct with the target gene, and the third group of mice (AAV eGFP) received the vector construct inserted without target gene.

Behavior was evaluated in the open field and forced swim tests six weeks after the introduction of vector constructs.

The mRNA levels of genes were determined using realTime PCR. The level of brain serotonin and their metabolite 5-HIAA were measured using HPLC (high-performance liquid chromatography).

Data were expressed as average values with an error of average $(\mathrm{x} \pm \mathrm{Sx})$. All groups were compared using singlefactor analysis of variance (one-way ANOVA) followed by multiple comparison using the Fisher post-hoc test.

\section{Results}

In mice that were injected with a construct with the target gene, an increase in the mRNA level of 5-HT7 receptor gene in the midbrain was detected, compared to animals of the AAV_eGFP $(p<0.001)$ and buffer $(p<0.001)$ groups. These data suggest that the viral construct introduced to the animal effectively serves as a vector and the plasmid with the target gene is actually transcribed in neurons.
One-way ANOVA showed the effect of 5-HT7 gene overexpression in the forced swim test $(p<0.05)$. There was a significant increase in the mobility of mice from the experimental group compared to the control group $(p<0.01)$ and the AAV_eGFP group $(\mathrm{p}<0.05)$, which indicates a decrease in level of depressive-like behavior in ASC mice with 5-HT7 gene overexpression.

The brain 5-HT level was not differed between all groups. In mice that received a viral construct with the target gene, an increase in the level of 5-HIAA in the cortex was detected, compared to animals from the AAV_eGFP group $(\mathrm{p}<0.01)$. Also, the significant increase in the 5-HIAA level in hippocampus was found in animals with 5-HT7 gene overexpression compared to the control group $(\mathrm{p}<0.001)$ and the AAV_eGFP group $(p<0.001)$. Moreover, the animals of the experimental group showed an increase in the serotonin metabolism index, defined as the ratio of 5-HIAA/serotonin, compared to the control group $(\mathrm{p}<0.01)$ and the AAV_eGFP group $(\mathrm{p}<0.05)$ in all the studied brain structures.

The data indicate that the 5-HT7 gene overexpression in midbrain led to more intense functioning of the brain serotonin system and this was accompanied by decrease in depressive-like behavior in ASC mice.

\section{ACKNOWLEDGMENT}

The study was supportet by Russian Science Foundation (grant number 19-15-00025).

\section{REFERENCES}

[1] J. G. Hensler (2012) Serotonin, In: S.T. Brady editor: Basic neurochemistry principles of molecular, cellular, and medical neurobiology. 8th ed. Academic. Waltham. MA.: 300-322.

[2] N. K. Popova, E. G. Ponimaskin, V. S. Naumenko (2015) Cross-talk between 5-HT1A and 5-HT7 receptors: role in the autoregulation of the brain serotonin system and in mechanism of antidepressants action. Russian Journal of Physiology. 101(11): 1270-1278.

[3] B. Dean (2003) The cortical serotonin 2A receptor and the pathology of schizophrenia: a likely accomplice. J. Neurochem. 85: 1-13.

[4] P. B. Hedlund (2009) The 5-HT7 receptor and disorders of the nervous system: an overview. Psychopharmacology (Berl). 206(3): 345-354. 